\title{
Radiation-Induced Large Vessel Cerebral Vasculopathy in Pediatric Patients with Brain Tumors Treated with Proton Radiotherapy
}

\author{
Stephen F. Kralik, MD ${ }^{1}$ \\ Chie-schin Shih, MD ${ }^{2}$ \\ Chang Y. Ho, $\mathrm{MD}^{1}$ \\ Whitney Finke, $\mathrm{MD}^{1}$ \\ Jeffrey Buchsbaum, $\mathrm{MD}^{3}$ \\ Gordon A. Watson, $\mathrm{MD}^{3}$ \\ ${ }^{1}$ Department of Radiology and Imaging Sciences, Indiana University School of Medicine, \\ Indianapolis, Indiana \\ ${ }^{2}$ Department of Pediatrics, Hematology/Oncology Section, Indiana University School of \\ Medicine, Indianapolis, Indiana \\ ${ }^{3}$ Department of Radiation Oncology, Indiana University School of Medicine, Indianapolis, \\ Indiana
}

Conflict of interest: None.

Funding: No source of funding was used for this research.

Correspondence to:

Stephen F. Kralik M.D.

Department of Radiology and Imaging Sciences, Indiana University School of Medicine,

714 N. Senate Avenue

Indianapolis, Indiana, 46202

Tel:1 (317) 963-7121

Fax: $1(317) 948-8383$

This is the author's manuscript of the article published in final edited form as:

Kralik, S. F., Shih, C., Ho, C. Y., Finke, W., Buchsbaum, J., \& Watson, G. A. (2017). Radiation-Induced Large Vessel Cerebral Vasculopathy in Pediatric Patients with Brain Tumors Treated with Proton Radiotherapy. International Journal of Radiation Oncology ${ }^{\star}$ Biology ${ }^{\star}$ Physics. https://doi.org/10.1016/j.ijrobp.2017.07.009 
E-mail: steve.kralik@gmail.com 


\title{
Radiation-Induced Large Vessel Cerebral Vasculopathy in Pediatric Patients with Brain Tumors Treated with Proton Radiotherapy
}

\author{
ABSTRACT \\ PURPOSE: The purpose of this research is to evaluate the incidence, time to development, \\ imaging patterns, risk factors, and clinical significance of large vessel cerebral vasculopathy in \\ pediatric patients with brain tumors treated with proton radiotherapy.
}

MATERIALS AND METHODS: A retrospective study was performed on 75 consecutive pediatric patients with primary brain tumors treated with proton radiotherapy. Radiation-induced large vessel cerebral vasculopathy (RLVCV) was defined as intracranial large vessel arterial stenosis or occlusion confirmed on MRA, CTA, and/or catheter angiography within an anatomic region with previous exposure to proton beam therapy and not present prior to radiotherapy. Clinical records were used to determine the incidence, timing, radiation dose to the large vessels, and clinical significance associated with the development of large vessel vasculopathy in these patients.

RESULTS: RLVCV was present in 5/75 (6.7\%) of patients and included tumor pathologies of craniopharyngioma (2), ATRT (1), medulloblastoma (1), and anaplastic astrocytoma (1). Median time from completion of radiotherapy to development was 1.5 years (mean 3.0 years; range 1.07.5 years). Neither mean age at time of radiotherapy (5.1 years) nor mean radiotherapy dose to the large vessels (54.5 Gy) were statistically significant risk factors. Four of the five patients with RLVCV presented with acute stroke, and demonstrated MRI evidence of acute infarcts in the expected vascular distributions. Angiography studies demonstrated collateral vessel formation in only two of the patients with RLVCV. No patients demonstrated acute hemorrhage or aneurysm. Two patients were treated with pial synangiomatosis surgery.

CONCLUSION: RLVCV can occur in pediatric patients with brain tumors treated with proton radiotherapy. Further studies are necessary to determine potential risk factors for large vessel vasculopathy with proton radiotherapy in comparison with conventional photon radiotherapy.

Keywords: Proton radiotherapy; vasculopathy; pediatric; brain

Abbreviations: ACA: anterior cerebral artery; ATRT: atypical teratoid rhabdoid tumor; CTA: CT Angiography; DWI: diffusion-weighted; FLAIR: fluid attenuated inversion recovery; GBM: glioblastoma multiforme; MCA: middle cerebral artery; MPRAGE: magnetization prepared rapid acquisition gradient echo; MRA: MR Angiography; PBT: proton beam radiotherapy; PCA: posterior cerebral artery; PNET: primitive neuroepithelial tumor; RLVCV: radiation induced large vessel cerebral vasculopathy; T1W: T1-weighted; T2W: T2-weighted; TSE: turbo spin echo 


\section{INTRODUCTION}

Radiotherapy is one of the primary therapies used to treat pediatric brain tumors. Potential adverse effects of radiotherapy on the brain include radiation necrosis, atrophy, gliosis, telangiectasia, cavernous malformations, and vasculopathy. Radiation-induced cerebral vasculopathy is relatively uncommon but has reported following conventional photon radiotherapy for pediatric brain tumors, most commonly gliomas, and medulloblastomas. ${ }^{1}$ Radiation-induced vasculopathy histologically demonstrates subintimal foam cells and myointimal proliferation, fibrous thickening and hyalinization of the subintima, thickening of the elastica, fibrous thickening of the adventitia, and loose connective tissue thickening occluding the lumen. ${ }^{2}$ A correlation between radiation dose and age at radiotherapy with development of radiation-induced vasculopathy has been previously reported. ${ }^{1,3}$ Although radiation-induced vasculopathy can be diagnosed on histopathology, the diagnosis is primarily made by radiological evidence of arterial stenosis or occlusion of a large intracranial vessel that was previously normal prior to radiotherapy.

Compared with conventional (photon) radiation therapy, proton beam radiotherapy (PBT) offers the advantages of the absence of an exit dose, a highly conformal dose distribution, and a reduced radiation dose to adjacent normal tissue. ${ }^{4}$ Therefore, potential benefits of PBT in patients with pediatric brain tumors may include the reduction of negative long-term effects of radiation, such as cognitive deficits, endocrine abnormalities, vascular abnormalities, and secondary malignancies. ${ }^{5} \mathrm{PBT}$ has been used increasingly to treat pediatric brain tumors, including craniopharyngiomas, ependymomas, germinomas, and medulloblastomas; however, radiation-induced large vessel cerebral vasculopathy (RLVCV) has been reported in only a few 
patients with PBT suggesting the complication is rare or yet to be fully evaluated in the literature. ${ }^{6-12}$

Therefore, we evaluated the incidence, timing, imaging patterns, risk factors, and clinical significance of RLVCV in a cohort of pediatric patients with brain tumors treated with PBT.

\section{MATERIALS AND METHODS}

Following institutional review board approval, a retrospective study was performed from March 2007 to December 2014 on 75 pediatric patients age < 18 with primary brain tumors who were treated with PBT and with radiation field covering the large intracranial arteries and who had clinical and MR imaging follow-up performed at our institution. Large cerebral arteries included the intracranial internal carotid artery (ICA), vertebral artery, basilar artery, middle cerebral artery (MCA) M1 segment, anterior cerebral artery (ACA) A1 segment, and posterior cerebral artery P1 segment. All patients were either those with a newly diagnosed primary brain tumor who were subsequently treated with PBT or those who had low-grade gliomas without prior radiation therapy who demonstrated tumor progression on chemotherapy necessitating treatment with PBT. Patients were excluded if there was any history of treatment with photon radiation therapy, including before PBT, concurrent with PBT, or after PBT. Patients were also excluded if there was a prior course of PBT. Patients without at least 6 months of clinical followup and MR imaging follow-up from the completion of PBT were excluded, including if death occurred before 6 months.

PBT treatment doses followed the standard of care in the United States at a Children's 
Oncology Group treatment center with a continuum of radiation doses, the majority of patients receiving between 50 to $60 \mathrm{~Gy}$ total. The radiation oncologist (J.C.B.) approved 3D image guidance treatment plans before every single field in real-time for every patient. Typical target volumes with PBT included gross tumor volume (any visible residual tumor and/or resection cavity) to a clinical target volume (area of concern) margin of $5 \mathrm{~mm}$; and the planning tumor volume (gross tumor volume plus clinical target volume) margin was set as $2 \mathrm{~mm}$ with a 5-mm margin when accounting for additional factors including smearing. All craniospinal radiation therapy was performed with PBT, not photon radiation therapy. The 3-D radiation dose distributions for each proton radiation plan were reviewed with attention to the relevant large intracranial vessels to confirm the large intracranial vessels were included in the radiation field and to determine the maximum radiation dose to the large intracranial arteries was recorded for all patients. In general, the median dose to the vertebral and basilar arteries were determined for posterior fossa tumors, and the median dose to the circle of Willis was calculated for supratentorial tumors.

Brain MRI consisted of imaging performed with 1.5T or 3T (Avanto and Verio; Siemens, Erlangen, Germany) MR imaging units with axial and sagittal T1-weighted (T1W) turbo spin echo (TSE) or magnetization prepared rapid acquisition gradient echo (MPRAGE), axial T2weighted (T2W) TSE, axial fluid attenuated inversion recovery (FLAIR), axial diffusionweighted (DWI), coronal T1W TSE postcontrast with fat saturation, and axial 3D T1W MPRAGE post contrast pulse sequences. T1W MPRAGE sequences were performed at $1 \mathrm{~mm}$ slice thickness while other sequences were at $4 \mathrm{~mm}$. Postcontrast imaging was performed in all patients after $0.1-\mathrm{mmol} / \mathrm{kg}$ intravenous administration of gadobenate dimeglumine (MultiHance; Bracco Diagnostics, Princeton, New Jersey). In the majority of patients, brain MRIs were 
performed approximately every 1-3 months for 2-3 years, then every 6-12 months with shorter or longer follow-up depending on clinical need to assess tumor stability, clinical symptoms, and/or other complications of chemoradiation such as radiation necrosis. MR Angiography (MRA) of the brain, was performed with axial 3D time-of-flight technique with $0.7 \mathrm{~mm}$ slice thickness and with rotating maximum intensity projection images of the anterior and posterior circulation. CT angiography (CTA) of the brain was performed with Philips iCT 256 slice CT scanner (Philips, Best, Netherlands) with $0.9 \mathrm{~mm}$ slice thickness following administration of Isovue-370 (Bracco Diagnostics, Princeton, New Jersey) intravenous contrast and multi-planar reconstructions and maximum intensity projection images were performed in the coronal and sagittal planes. Catheter angiography was performed with Toshiba biplane (Toshiba Medical Systems, Tokyo, Japan) digital subtraction angiography with multiplanar views of the right and left internal and external carotid arteries and the vertebral arteries following intravenous contrast injection.

Diagnosis of RLVCV was made if stenosis or occlusion of a large cerebral vessel was not present on imaging prior to PBT but developed on imaging following PBT, and was confirmed with either MRA, CTA, or catheter angiography. Diagnosis of RLVCV was not dependent on clinical manifestation of stroke. A fellowship-trained, board-certified neuroradiologist (S.F.K.) with certificate of added qualification in neuroradiology independently reviewed all MRIs, MRAs, CTAs, and catheter angiography studies before and after PBT. Attention on all MRI studies was made to the large vessels on $\mathrm{T} 2 \mathrm{~W}$ and $\mathrm{T} 1 \mathrm{~W}$ post contrast MPRAGE sequences in all patients as a means to assess arterial stenosis. Assessment of arterial stenosis or occlusion was preformed by visual comparison to normal segments of the vessel or contralateral vessel when appropriate. When stenosis was present, the arterial stenosis was calculated based on the ratio of the arterial diameter of the narrowed segment to the arterial diameter of the normal proximal 
segment and defined as mild for $<25 \%$ stenosis, moderate for $25-75 \%$ stenosis, and severe for $>$ $75 \%$ stenosis. Evidence of acute intracranial infarcts or hemorrhage on MRI, and aneurysm(s) on MRA, CTA, or catheter angiography were also recorded. Clinical data obtained from the electronic medical record included patient age at time of PBT, patient gender, tumor pathology (with exception of some brainstem tumors which did not have pathology specimen), tumor location, total cranial radiation dose, timing from completion of PBT to imaging diagnosis of large vessel vasculopathy, treatment of vasculopathy, and clinical symptoms related to large vessel vasculopathy.

Statistical analysis of clinical risk factors was performed by using a Fisher exact test and unpaired t test where appropriate. Statistics were performed using Graphpad Prism 7 Statistical Software (La Jolla, CA; http://www.graphpad.com; 2016) with $\mathrm{p}<0.05$ considered statistically significant. A Kaplan-Meier curve for probability of not having RLVCV was calculated using Vassarstats (http://vassarstats.net) and probability with 95\% confidence intervals (CI) were calculated.

\section{RESULTS}

Patient characteristics are listed in Table 1. A total of 5/75 (6.7\%) of patients developed RLVCV. The median follow-up duration was 4.3 years (mean 4.2 years, range 0.6-9.6 years). Among the four patients with RLCVC, intracranial pathology consisted of craniopharyngioma (2), medulloblastoma (1), anaplastic astrocytoma (1), and ATRT (1). Representative examples of patients with RLCVC are seen in figures 1 and 2. The mean age at time of PBT for patients who 
developed vasculopathy was 5.1 years compared to 8.0 years for patients without vasculopathy $(\mathrm{p}=0.17)$. The median time to development of large vessel vasculopathy was 1.5 years (mean 3.0 years; range 1.0-7.5 years). A Kaplan Meier curve demonstrating the probability of development of RLVCV is seen in figure 3. The probability of not having RLVCV was 96\% (88-99\%, $95 \% \mathrm{CI})$ at 3 years, $95 \%(86-98 \%, 95 \% \mathrm{CI})$ at 4 years, and $95 \%(85-98 \%, 95 \% \mathrm{CI})$ at 5 years. The mean radiation dose to the large cerebral arteries of patients at time of radiotherapy who developed vasculopathy was 54.5 Gy compared to 50.6 Gy for patients without vasculopathy $(\mathrm{p}=0.30)$

Prior to PBT, MRA was performed in five patients because the patient's initial tumor presented as a hemorrhagic mass requiring assessment for an arteriovenous malformation. None of these MRAs demonstrated large intracranial artery stenosis. Prior to PBT, MRIs of the brain also did not demonstrate evidence of large intracranial artery stenosis except in one patient who had a suprasellar astrocytoma encasing the left ICA, MCA, and ACA that resulted in moderate arterial stenosis of these vessels.

After PBT, eleven patients had MRA or CTA in conjunction with MRI, of which five were diagnosed with RLVCV based on presence of arterial stenosis. Details of patients with RLVCV are found in Table 2. Vasculopathy imaging findings included three patients with unilateral vasculopathy (left ICA occlusion; right ICA, MCA, and ACA stenosis; segmental basilar artery occlusion), and two patients with bilateral vasculopathy (bilateral vertebral artery stenosis; right ICA and left ACA stenosis). Three of the the five patients had no visible collateral vessels on angiography studies. Four of the five patients presented clinically with acute stroke manifested as acute hemiparesis and had acute intracranial infarct(s) identified as MRI evidence of diffusion restriction in the distribution of the large vessel vasculopathy. Infarct 
distribution pattern included two patients with MCA and ACA territory infarcts involving nearly the entire vascular territories, one patient with MCA territory infarct involving approximately $25 \%$ of the MCA territory, and one patient with a unilateral pontine lacunar infarct involving less than $25 \%$ of the pons. The fifth patient who had a segmental occlusion of the basilar artery had chronic unilateral weakness which preceded radiation therapy and was attributed to the tumor involving the internal capsule and cerebral peduncle, but no clinical episode of stroke nor MRI evidence of acute or chronic infarct.

Two patients had hypercoagulopathy laboratory testing with one patient demonstrating low anti-thrombin III and low protein $\mathrm{C}$ that was attributed to the acute phase of stroke, and one patient had high anti-thrombin III and low protein C. None of the patients had cholesterol laboratory testing. Two patients underwent pial synangiosis surgery. Three patients demonstrated residual hemiparesis of the affected side on last follow-up ranging from 26 months to 7 years. The patient with the pontine infarct was lost to follow-up. One patient died 26 months after the diagnosis of radiation-induced vasculopathy secondary to tumor recurrence.

None of the patients with LVCV had acute intracranial hemorrhage, intracranial aneurysm, or neurofibromatosis type-1. Among patients with LVCV, none were retrospectively identified on preceding MRI T2W or post contrast T1W MPRAGE sequences that were performed at a range of 2-6 months previously. Among patients without LVCV, none were identified to have clinically asymptomatic arterial stenosis on $\mathrm{T} 2 \mathrm{~W}$ or post contrast $\mathrm{T} 1 \mathrm{~W}$ MPRAGE.

\section{DISCUSSION}


To our knowledge, this study is the largest series of RLVCV associated with PBT. In this study the crude incidence of RLVCV was approximately $6.7 \%$, median time to development was 1.5 years (mean 3.0 years; range 1-7.5 years), and median age at time of PBT was 5.1 years. In comparison, reported photon radiotherapy large vessel vasculopathy demonstrates median time to development of 5 years (range 0.3-27 years), and median age at time of radiotherapy of 4.83 years. ${ }^{1}$ The incidence of vasculopathy in our study was less than that reported by Omura et al who reported large vessel vasculopathy in 6/32 (19\%) of pediatric patients with primary brain tumors in whom photon radiation fields included the circle of Willis and large intracranial vessels although this study had median follow up duration of 5.2 years. ${ }^{3}$ Further studies are necessary to determine whether this difference in incidence reflects a reduction in large vessel vasculopathy with PBT compared to photon radiotherapy. At this time there is no reason to anticipate a difference in toxicity, including RLVCV, to normal tissues within a radiation treatment volume receiving a given dose, whether that dose was delivered by photon or PBT. We cannot assess whether the risk of RLVCV outside the target volume may be lower with PBT versus photon therapy, although the dose reduction to non-target tissues achieved by PBT may reduce the risk.

The vasculature of pediatric patients is suspected to be more susceptible to effects of radiotherapy. ${ }^{13}$ In this study we are unable to demonstrate radiotherapy dose and age are risk factors for large vessel vasculopathy from PBT, although these results are likely due to the low incidence and our study may be helpful as more studies report results on radiation-induced vasculopathy with PBT. Omura et al performed a single institution evaluation of radiation induced large vessel vasculopathy and were able to demonstrate that increasing radiation dose was a risk factor but similarly could not determine if age was a risk factor, likely due to the low 
incidence of large vessel vasculopathy at their single institution similar to our study. ${ }^{3}$ Wang et al, however, evaluated 77 reports of large vessel vasculopathy in pediatric patients with brain tumors treated with conventional radiotherapy and were able to demonstrate that younger age was a risk factor. ${ }^{1}$ We suspect that younger age and higher radiation dose remain risk factors for development of large vessel vasculopathy with PBT based on data from conventional radiotherapy, but further reports of patients with large vessel vasculopathy from PBT will be necessary to reach this conclusion.

Radiation-induced vasculopathy has been reported to result in acute ischemic infarct, intraparenchymal hemorrhage, and aneurysm. ${ }^{14-18}$ This study demonstrates that a majority of patients with RLVCV will present with acute stroke, often with significant permanent neurological deficit, and may require surgical treatment to promote collateral vessel formation. Campen et al reported a 100-fold increase in risk of transient ischemic attack or stroke compared to the general population, a median time of 4.9 years from initial radiation to time of stroke, and an increased risk of stroke in patients with radiation therapy to the circle of Willis. ${ }^{14}$ Similarly, El-Fayech et al demonstrated that radiation doses of $10 \mathrm{~Gy}$ or more to the circle of Willis was associated with a cumulative stroke incidence of $11.3 \%$ compared to an expected incidence of $1 \%$ in the general population. ${ }^{19}$ Our study, similar to other studies, is unable to determine what additional genetic or metabolic factors may contribute to the vasculopathy. Lastly, although RLVCV and radiation-induced Moyamoya are generally considered synonymous, we chose the term RLVCV rather than radiation-induced Moyamoya for purposes of consistency with the majority of the literature on this subject, and because the majority of these patients do not exhibit significant collateral vessel formation like patients with Moyamoya vasculopathy. The lack of extensive collateral vessel formation suggests the stenosis may progress more rapidly than 
Moyamoya and likely accounts for the large areas of infarct encountered in some patients. This may also account for our inability to identify progressive stenosis occurring within the large arteries on MRIs prior to acute presentation of stroke and arterial stenosis or occlusion.

As the number of PBT centers increases, it is important that physicians are aware of the potential for RLVCV in pediatric patients treated with PBT, particularly tumors near the circle of Willis. Neurological signs and symptoms of transient ischemic attacks may precede an acute stroke and may indicate need for vascular imaging in these patients. Although none of the patients demonstrated arterial stenosis on the MRI performed between 2-6 months preceding the diagnosis, we recommend that radiologists should still assess the intracranial vasculature on all patients treated with radiotherapy, particularly those tumors near the circle of Willis and recommend vascular imaging if there is suspicion of arterial stenosis on conventional MRI. It is uncertain if advanced imaging techniques such as MR perfusion or arterial wall imaging may help identify patients prior to development of arterial stenosis or be helpful to identify areas at risk for acute infarct. ${ }^{20}$

\section{LIMITATIONS:}

Because current clinical standard of care for these patients does not include routine performance of CTA, MRA, or catheter angiography, we acknowledge the potential limitation for determining the incidence and timing of RLVCV. Despite our efforts to review MRI sequences that best demonstrate the large intracranial arteries such as $\mathrm{T} 2 \mathrm{~W}$ and $\mathrm{T} 1 \mathrm{~W}$ post contrast MPRAGE, we were unable to identify large vessel stenosis on the MRIs obtained months in advance among patients with RLVCV who presented with acute stroke. Consequently, we are unable to conclude at this time that surveillance CTA or MRA should be performed in 
these patients. Lastly, the RLVCV reported herein was not confirmed by histology, however, current clinical standard of care does not include arterial biopsy such that the diagnosis of RLVCV based on clinical and radiological data more closely reflects current clinical practice.

Although this study represents the largest report of pediatric patients with RLVCV following PBT, this study represents a single institution experience and highlights the need for future investigations using multi-center collaboration with greater number of patients and expanded follow up duration to further understand the similarities and differences of PBT compared photon radiotherapy with respect to RLVCV. Incidence of RLVCV will likely be affected by follow up duration. In this research, our follow-up duration was approximately 4 years which may still underestimate the incidence of RLVCV, however, we believe this does not detract from the importance of this research in building a foundation for additional research into RLVCV with proton radiotherapy and bringing greater clinical attention to this complication. Lastly, genetic and/or metabolic factors that may increase the risk of RLVCV to pediatric patients remain unknown and require further investigation.

\section{CONCLUSION}

Radiation-induced large vessel cerebral vasculopathy may occur in pediatric patients with brain tumors treated with PBT. With longer survival of pediatric patients with brain tumors, it is important for physicians to recognize the potential for RLVCV in pediatric patients with brain tumors treated with PBT. Further studies are necessary to advance the understanding of risk factors for development of RLVCV associated with PBT. 


\section{REFERENCES:}

1. Wang C, Roberts KB, Bindra RS, et al. Delayed cerebral vasculopathy following cranial radiation therapy for pediatric tumors. Pediatr Neurol. 2014 Jun;50(6):549-56.

2. Brant-Zawadzki M, Anderson M, DeArmond SJ, et al. Radiation-induced large intracranial vessel occlusive vasculopathy. AJR Am J Roentgenol. 1980 Jan;134(1):51-5.

3. Omura M1, Aida N, Sekido K, Kakehi M, Matsubara S. Large intracranial vessel occlusive vasculopathy after radiation therapy in children: clinical features and usefulness of magnetic resonance imaging. Int J Radiat Oncol Biol Phys. 1997 May 1;38(2):241-9.

4. Levin WP, Kooy H, Loeffler JS, et al. Proton beam therapy. Br J Cancer 2005;93:849 54.

5. Boehling NS, Grosshans DR, Bluett JB, et al. Dosimetric comparison of threedimensional conformal proton radiotherapy, intensity-modulated proton therapy, and intensity-modulated radiotherapy for treatment of pediatric craniopharyngiomas. Int $\mathbf{J}$ Radiat Oncol Biol Phys 2012;82:643-52.

6. Howell RM, Giebeler A, Koontz-Raisig W, et al. Comparison of therapeutic dosimetric data from passively scattered proton and photon craniospinal irradiations for medulloblastoma. Radiat Oncol 2012;7:116.

7. MacDonald SM, Safai S, Trofimov A, et al. Proton radiotherapy for childhood ependymoma: initial clinical outcomes and dose comparisons. Int J Radiat Oncol Biol Phys 2008;71:979-86.

8. Jimenez RB, Sethi R, Depauw N, et al. Proton radiation therapy for pediatric medulloblastoma and supratentorial primitive neuroectodermal tumors: outcomes for 
very young children treated with upfront chemotherapy. Int J Radiat Oncol Biol Phys 2013;87:120-26.

9. MacDonald SM, Trofimov A, Safai S, et al. Proton radiotherapy for pediatric central nervous system germ cell tumors: early clinical outcomes. Int J Radiat Oncol Biol Phys 2011;79:121-29.

10. Zwagerman NT, Foster K, Jakacki R, et al. The development of Moyamoya syndrome after proton beam therapy. Pediatr Blood Cancer 2014 Aug;61(18):1490-2.

11. Hug EB, Muenter MW, Archambeau JO, et al. Conformal proton radiation therapy for pediatric low-grade astrocytomas. Strahlenther Onkol. 2002 Jan;178(1):10-7

12. Reynolds MR, Haydon DH, Caird J, et al. Radiation-Induced Moyamoya Syndrome after Proton Beam Therapy in the Pediatric Patient: A Case Series. Pediatr Neurosurg. 2016;51(6)297-301.

13. Rudoltz MS, Regine WF, Langston JW, et al. Multiple causes of cerebrovascular events in children with tumors of the parasellar region. J Neuro-oncol. 1998;37:251-261.

14. Campen CJ, Kranick SM, Kasner SE, et al. Cranial irradiation increases risk of stroke in pediatric brain tumor survivors. Stroke. 2012;43:3035-3040.

15. Mueller S, Fullerton HJ, Stratton K, et al. Radiation, atherosclerotic risk factors, and stroke risk in survivors of pediatric cancer: a report from the Childhood Cancer Survivor Study. Int J Radiat Oncol Biol Phys. 2013;86:649-655.

16. Matsumoto H, Minami H, Yamaura I, Yoshida Y. Radiation-induced cerebral aneurysm treated with endovascular coil embolization. A case report. Interv Neuroradiol. 2014 JulAug;20(4):448-53.

17. Azzarelli B, Moore J, Gilmor R, et al. Multiple fusiform intracranial aneurysms following 
curative radiation therapy for suprasellar germinoma. Case report. J Neurosurg. $1984 ; 61: 1141-1145$.

18. Jensen FK, Wagner A. Intracranial aneurysm following radiation therapy for medulloblastoma. A case report and review of the literature. Acta Radiol. 1997;38:37-42.

19. El-Fayech C, Haddy N, Allodji RS, et al. Cerebrovascular Diseases in Childhood Cancer Survivors: Role of the Radiation Dose to the Willis Circle Arteries. Int J Radiat Oncol Biol Phys. 2017 Feb 1;97(2):278-286.

20. Li M, Wu SW, Xu WH. High-resolution MRI of radiation-induced intracranial vasculopathy. Neurology. 2015 Feb 10;84(6):631. 
Figure 1. A 6-year-old with history of craniopharyngioma treated with proton radiotherapy. Sagittal T1W post contrast (A) image demonstrates the craniopharyngioma in the sella and suprasellar cistern (arrow) prior to radiotherapy. Sagittal radiation treatment image (B) demonstrate the dose lines of the proton beam treatment plan shown as percentages of the prescription dose (54 Gy). Axial DWI (C) image demonstrates a large region of hyperintensity in the right cerebral hemisphere consistent with an acute infarct involving the right ACA and MCA territories. Sagittal digital subtraction angiography (D) image following injection of the right ICA demonstrates severe stenosis of the right ICA (arrow) confirming the diagnosis of RLVCV. Digital subtraction angiography following injection of the left ICA (not shown) also demonstrated severe stenosis of the left ACA A1 segment with leptomeningeal collaterals providing retrograde filling of distal ACA branches.

Figure 2. An 18-month-old with history of ATRT treated with surgical resection, chemotherapy and proton radiotherapy. Axial DWI (A) image demonstrates a large region of hyperintensity consistent with an acute infarct in the left MCA distribution. Coronal CT Angiography (B) image demonstrates occlusion of the left supraclinoid ICA (arrow), decreased caliber of the left cavernous ICA segment, lack of filling of the left MCA, however both ACA vessels filling due to supply through the anterior communicating artery. Sagittal digital subtraction angiography (C) image following injection of the left ICA demonstrates severe stenosis at the origin of the posterior communicating artery and occlusion of the left ICA (arrow) just past the anterior choroidal artery, lack of filling of the left ACA and MCA vessels, and lack of lenticulostriate collateral vessels.

Figure 3. Kaplan-Meier curve demonstrating probability of radiation-induced large vessel cerebral vasculopathy over time. 
Table 1: Patient Characteristics

Category

Age

Gender

Tumor Pathology

Tumor Location

Total cranial radiation dose

\section{Characteristics}

Mean 7.9 years (range 1.5-18 years)

Male/Female 45:30

Medulloblastoma/PNET 25

Craniopharyngioma 14

Pilocytic/Pilomyxoid astrocytoma 10

Germinoma 6

Brainstem glioma 6

Ependymoma 4

ATRT 3

GBM/Anaplastic Astrocytoma 3

Mature teratoma 2

Pineoblastoma 1

Pituitary adenoma 1

Supratentorial 38

- Sella/Suprasellar/Optic Pathway 24

- Cerebral Hemisphere 7

- Pineal 7

Infratentorial 35

-Cerebellar $/ 4^{\text {th }}$ ventricle 27

-Brainstem 8

Multifocal 2

Mean 53.7 Gy (range 30-59.4 Gy) 
Table 2: Description of pediatric patients with radiation-induced large vessel vasculopathy

\begin{tabular}{|c|c|c|c|c|c|}
\hline Patient & 1 & 2 & 3 & 4 & 5 \\
\hline $\begin{array}{l}\text { Age at time } \\
\text { of } \\
\text { radiotherapy }\end{array}$ & 6 years & 1.5 years & 7 years & 8 years & 3 years \\
\hline $\begin{array}{l}\text { Time to } \\
\text { development }\end{array}$ & 3.8 years & 1 year & 7.5 years & 1 year & 1.5 years \\
\hline $\begin{array}{l}\text { Radiation } \\
\text { dose to large } \\
\text { arteries }\end{array}$ & $54 \mathrm{~Gy}$ & $54.6 \mathrm{~Gy}$ & $55.8 \mathrm{~Gy}$ & $54 \mathrm{~Gy}$ & $54 \mathrm{~Gy}$ \\
\hline Pathology & Craniopharyngioma & ATRT & Medulloblastoma & Craniopharyngioma & Anaplastic astrocytoma \\
\hline Location & $\begin{array}{l}\text { Sella and suprasellar } \\
\text { cistern }\end{array}$ & $\begin{array}{l}\text { Multifocal } \\
\text { supratentorial and } \\
\text { infratentorial } \\
\text { tumor foci }\end{array}$ & Cerebellum & Suprasellar cistern & $\begin{array}{l}\text { Multispatial: thalamus, } \\
\text { right cerebral peduncle, } \\
\text { optic chiasm, right temporal } \\
\text { lobe }\end{array}$ \\
\hline Presentation & $\begin{array}{l}\text { Acute right } \mathrm{MCA} \text { and } \\
\text { right ACA infarct }\end{array}$ & $\begin{array}{l}\text { Acute left MCA } \\
\text { infarct }\end{array}$ & $\begin{array}{l}\text { Acute pontine } \\
\text { infarct }\end{array}$ & $\begin{array}{l}\text { Acute right } \mathrm{MCA} \\
\text { and } \mathrm{ACA} \text { infarcts }\end{array}$ & No acute or chronic infarct \\
\hline $\begin{array}{l}\text { Vascular } \\
\text { Imaging } \\
\text { findings }\end{array}$ & $\begin{array}{l}\text { Right ICA stenosis } \\
\text { (severe) and left ACA } \\
\text { stenosis (severe); } \\
\text { collateral vessels } \\
\text { reconstitute the distal } \\
\text { ACA and small number } \\
\text { of collaterals from the } \\
\text { right middle meningeal } \\
\text { artery }\end{array}$ & $\begin{array}{l}\text { Left ICA } \\
\text { occlusion, no } \\
\text { collaterals }\end{array}$ & $\begin{array}{l}\text { Bilateral vertebral } \\
\text { artery stenosis } \\
\text { (moderate), no } \\
\text { collaterals }\end{array}$ & $\begin{array}{l}\text { Right ICA, MCA, } \\
\text { and ACA stenosis } \\
\text { (severe), no } \\
\text { collaterals }\end{array}$ & $\begin{array}{l}\text { Superior basilar artery } \\
\text { occlusion with collaterals }\end{array}$ \\
\hline Treatment & $\begin{array}{c}\text { Bilateral pial } \\
\text { synangiomatosis }\end{array}$ & $\begin{array}{l}\text { Left sided pial } \\
\text { synangiomatosis }\end{array}$ & None & None & None \\
\hline
\end{tabular}




\section{ACCEPTED MANUSCRIPT}
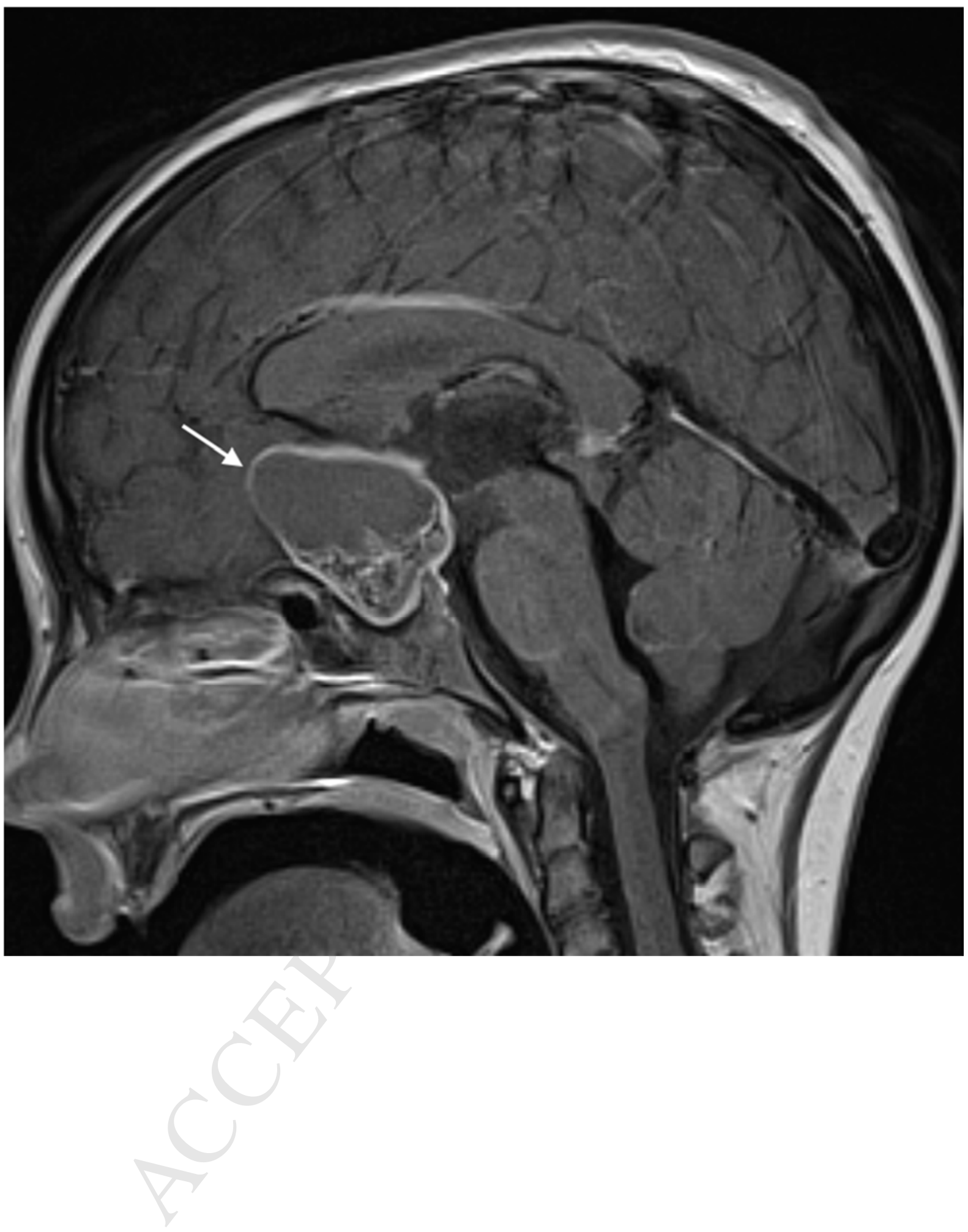


\section{ACCEPTED MANUSCRIPT}

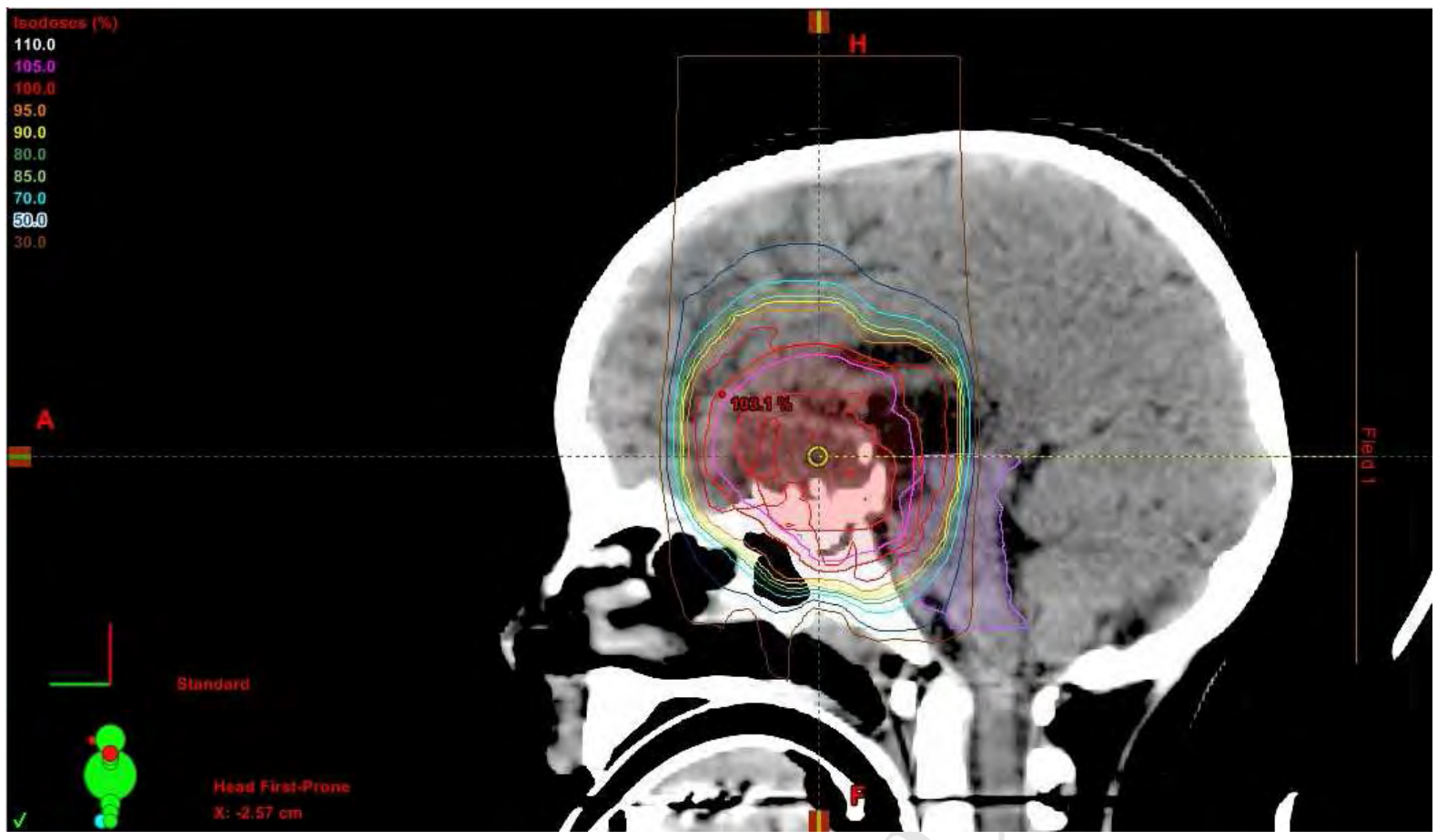




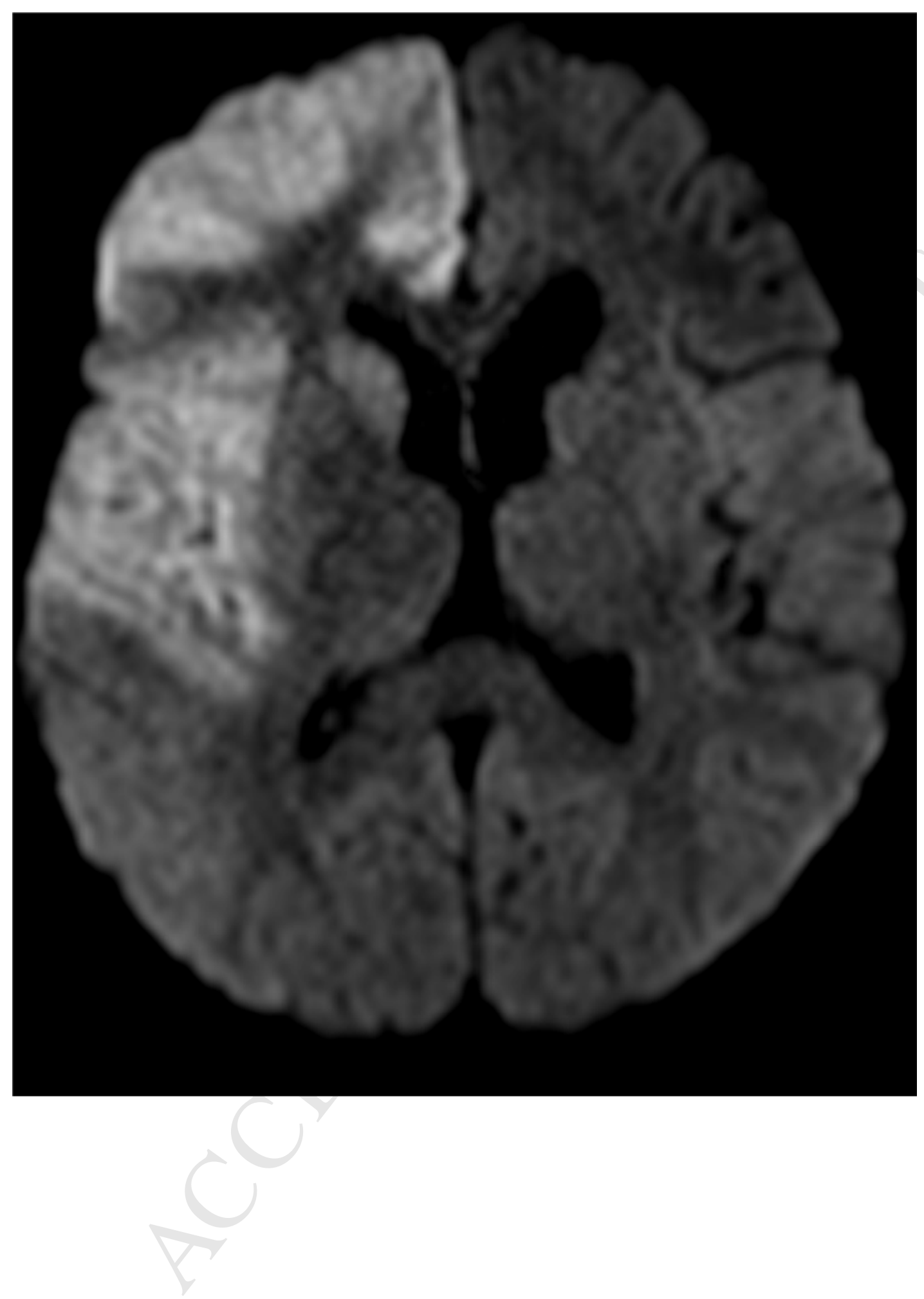




\section{ACCEPTED MANUSCRIPT}
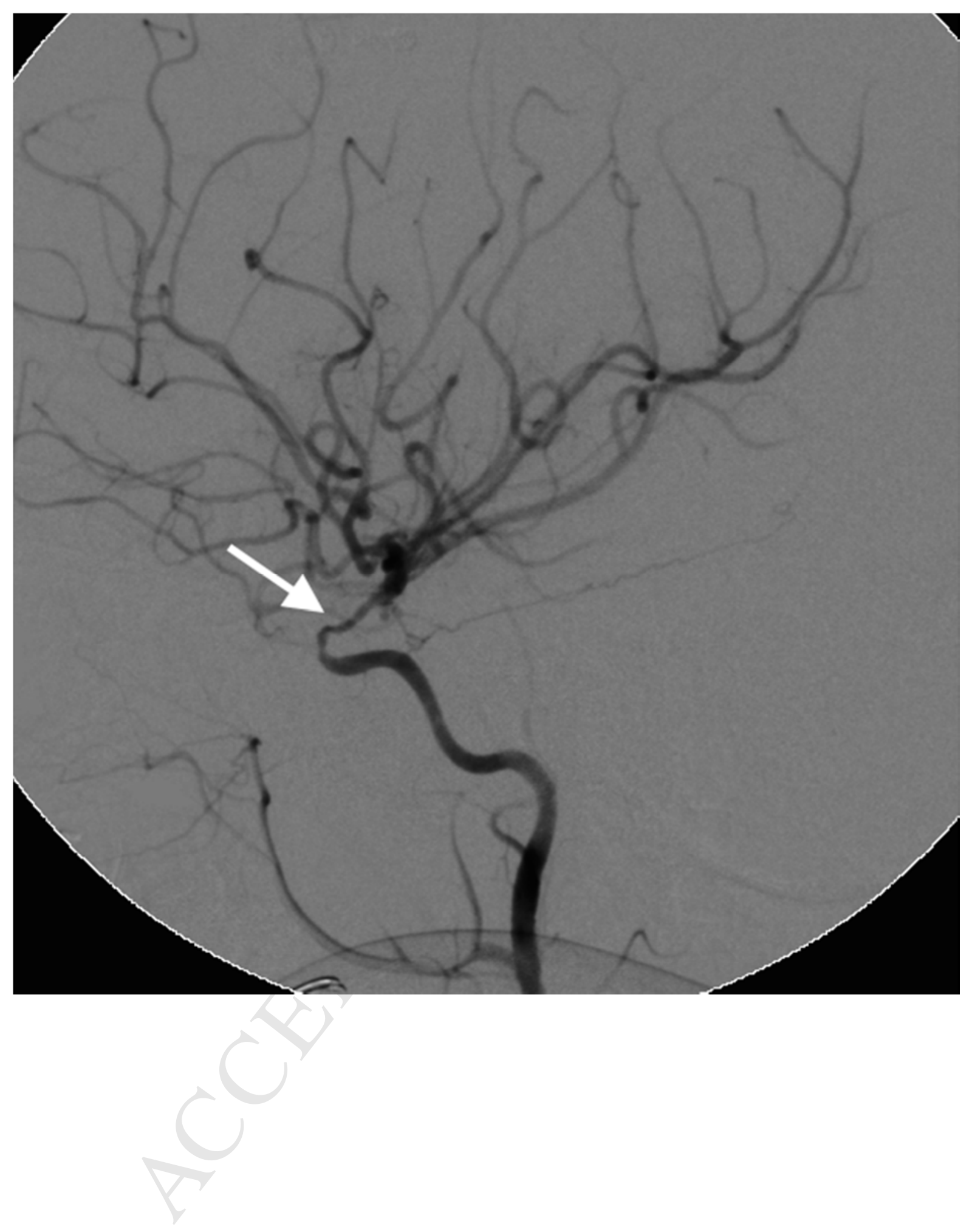


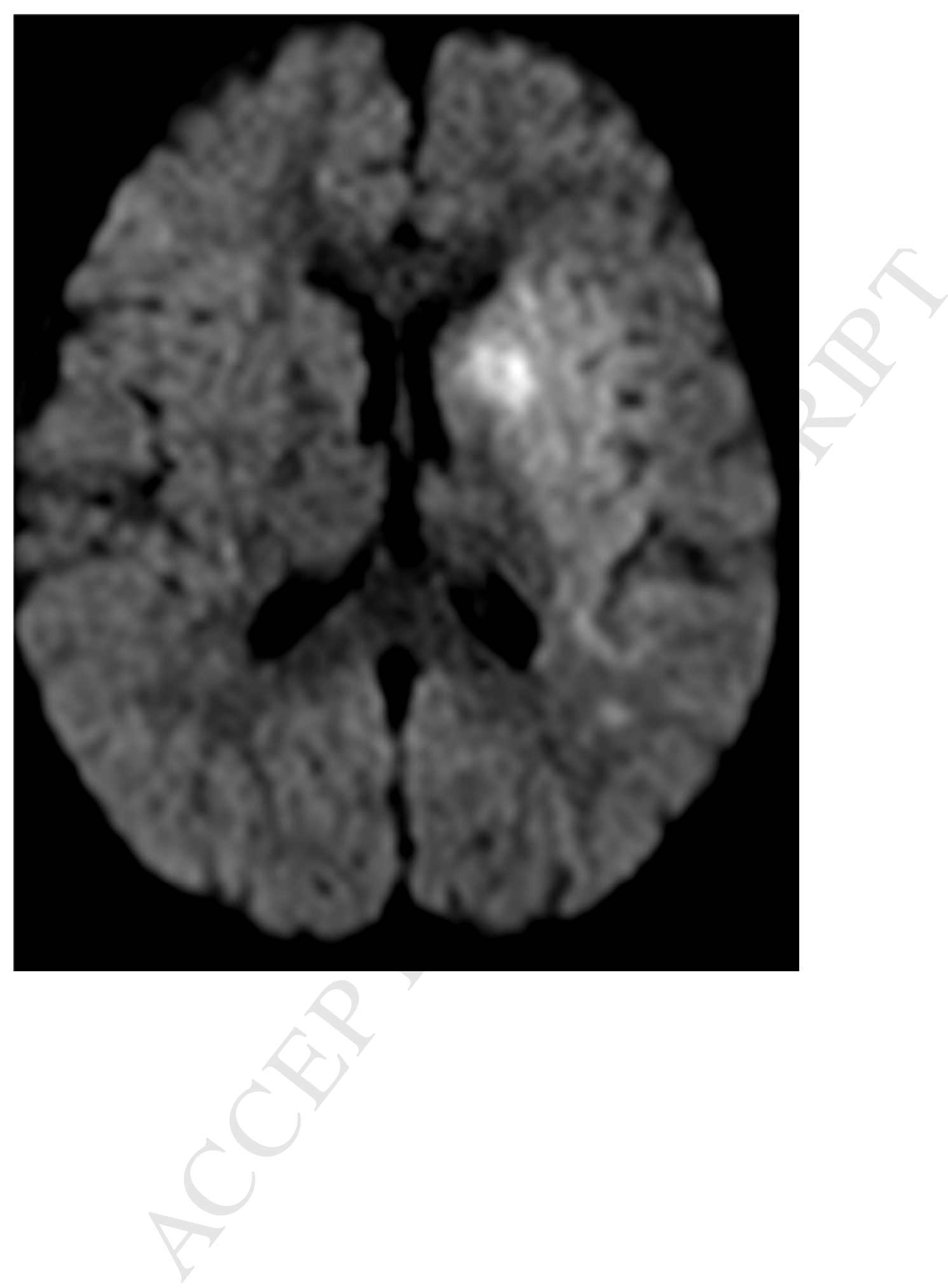




\section{ACCEPTED MANUSCRIPT}
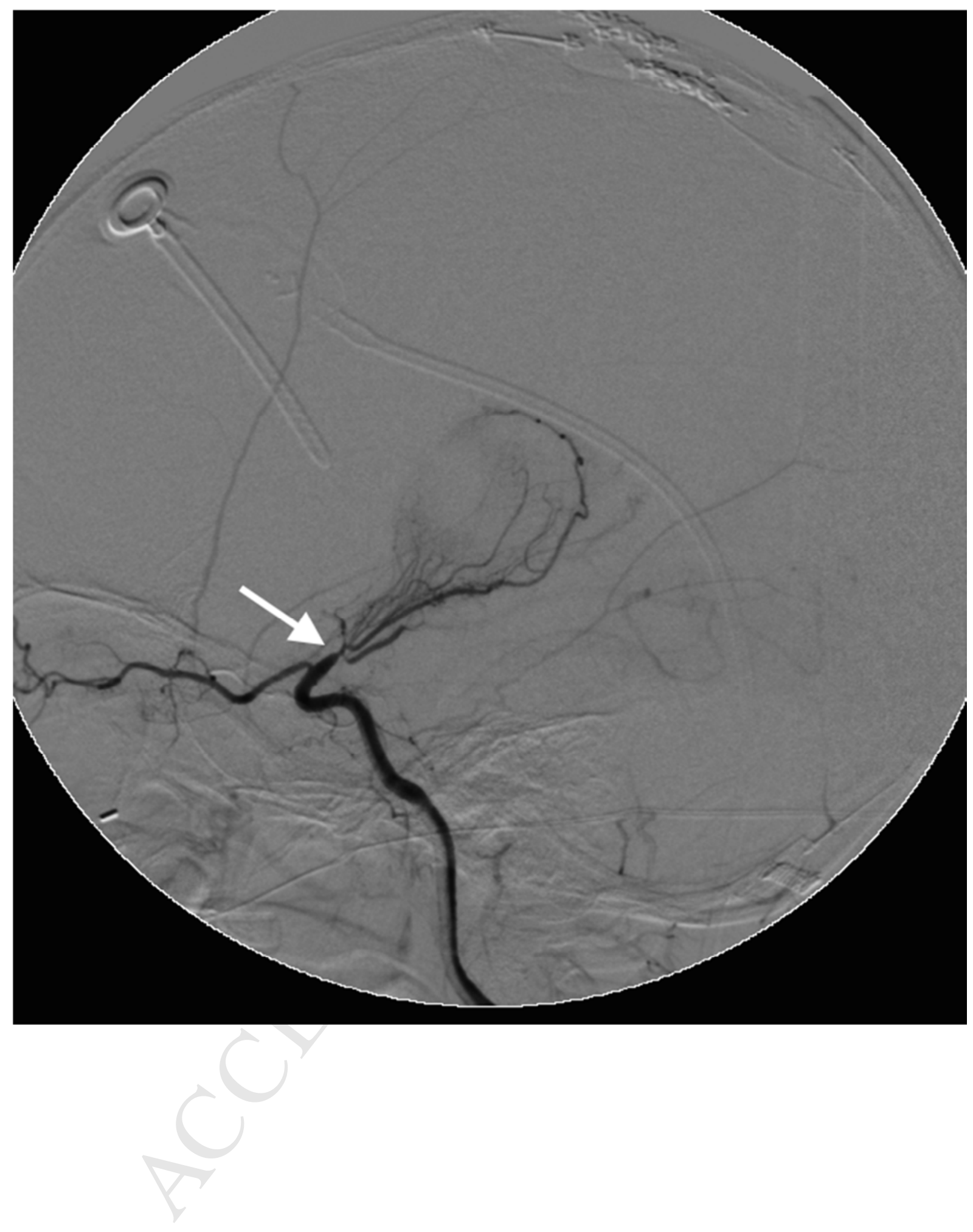


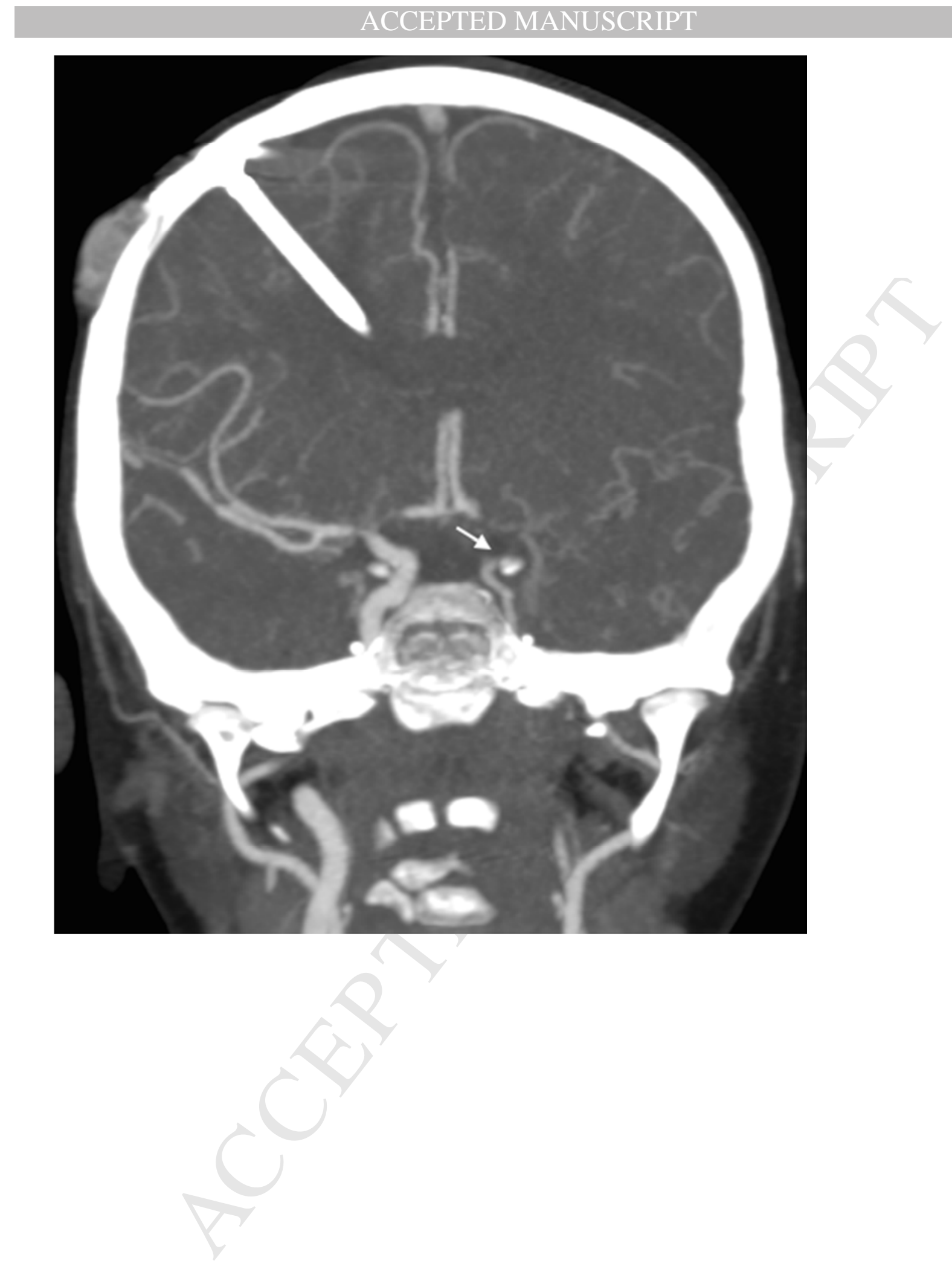




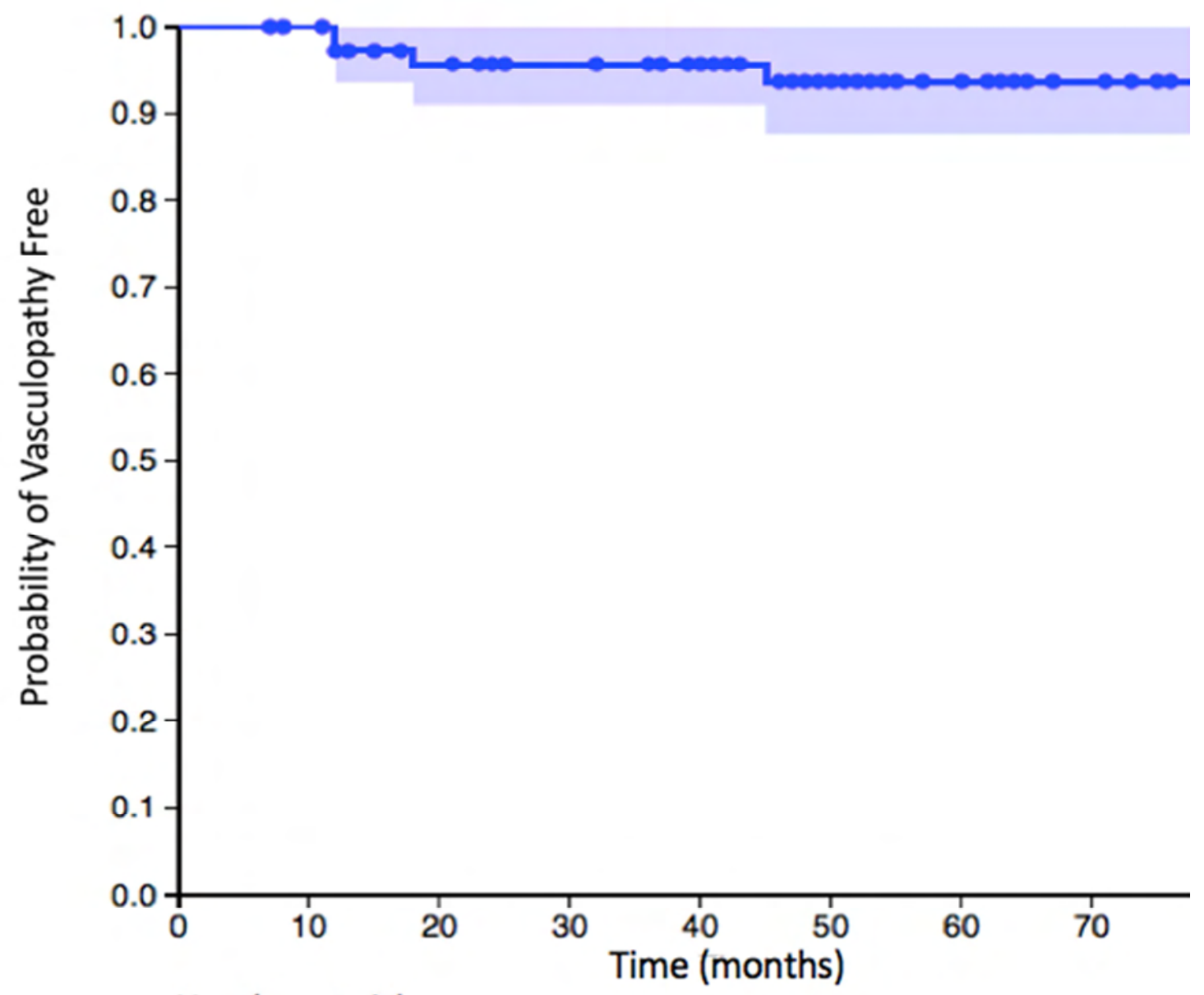

Number at risk

75

62

$58 \quad 53$

53

36

22 


\section{SUMMARY:}

This is the largest study to report radiation-induced large vessel cerebral vasculopathy in pediatric patients with primary brain tumors treated with proton radiotherapy. This complication can lead to stroke and associated permanent neurological disability. This research adds to the relative limited knowledge of radiation-induced large vessel cerebral vasculopathy, establishes an estimate for the timing and incidence of this complication, and may be used in future research for comparison to conventional radiotherapy. 